\title{
Genome dynamics of multidrug-resistant Acinetobacter baumannii during infection and treatment
}

\author{
Meredith S. Wright ${ }^{1}$, Alina lovleva ${ }^{2}$, Michael R. Jacobs ${ }^{3,4}$, Robert A. Bonomo ${ }^{5,6}$ and Mark D. Adams ${ }^{1 *}$
}

\begin{abstract}
Background: Limited treatment options are available for patients infected with multidrug (MDR)- or pan-drug (PDR)resistant bacterial pathogens, resulting in infections that can persist for weeks or months. In order to better understand transmission and evolutionary dynamics of MDR Acinetobacter baumannii (Ab) during long-term infection, we analyzed genomes from a series of isolates from individual patients at isolate-specific, patient-specific, and population levels.

Methods: Whole genome analysis of longitudinal isolates (range 2-10 isolates per patient spanning 0-829 days) from 40 patients included detection of single-nucleotide variants (SNVs), insertion sequence (IS) mapping, and gene content changes.

Results: Phylogenetic analysis revealed that a significant fraction of apparently persistent infections are in fact due to re-infection with new strains. SNVs primarily resulted in protein coding changes, and IS events primarily interrupted genes or were in an orientation such that the adjacent gene would be over-expressed. Mutations acquired during infection were over-represented in transcriptional regulators, notably pmrAB and adeRS, which can mediate resistance to the last line therapies colistin and tigecycline, respectively, as well as transporters, surface structures, and iron acquisition genes.

Conclusions: Most SNVs and IS events were isolate-specific indicating these mutations did not become fixed on the time scale investigated, yet over-representation of independent mutations in some genes or functional categories suggests that they are under selective pressure. Genome analysis at the population-level suggests that gene transfer including recombination also contributes to Ab evolutionary dynamics. These findings provide important insight into the transmission dynamics of $A b$ and the identification of patients with repeat infections has implications for infection control programs targeted to this pathogen.
\end{abstract}

\section{Background}

Bacterial pathogens resistant to multiple classes of antibiotics are increasingly detected in clinical settings, and patients with multidrug-resistant (MDR) infections have limited treatment options. This occurrence can translate into prolonged infections with increased morbidity and mortality $[1,2]$. Analysis of MDR strains isolated over time from individual patients during infection and treatment can reveal important adaptations to the host environment [3-5]. The most extensive work to date has characterized longitudinal genome changes in cystic fibrosis patients infected with Pseudomonas aeruginosa and Burkholderia

\footnotetext{
* Correspondence: madams@jcvi.org

${ }^{1}$ The J. Craig Venter Institute, La Jolla, CA, USA

Full list of author information is available at the end of the article
}

species, where these studies found that mutations were enriched in genes related to host adaptation and antibiotic resistance, and observed the co-existence of multiple lineages in the same host [6-10]. Patients with cystic fibrosis experience long-term infections, often spanning many years. Fewer studies have examined genomic changes in the context of acute infections that persevere during hospitalization, nor have longitudinal genomic changes within the context of a broader population structure been assessed.

In the last decade, Acinetobacter baumannii (Ab), one of the ESKAPE pathogens, emerged to become a predominant cause of nosocomial infections [11-14]. This is in part due to a remarkable rise in the frequency of MDR (resistant to at least three classes of antibiotic) and extreme drug 
resistant (XDR; resistant to all antibiotics except tigecycline or colistin) infections [12-14]. Notably, infections with carbapenem-resistant MDR and XDR strains are associated with longer hospitalization, greater economic costs, and higher mortality vs. carbapenem-susceptible strains [15-23].

Increased antibiotic resistance in $\mathrm{Ab}$ has been primarily driven by acquisition of resistance determinants encoded on mobile genetic elements and activation of intrinsic resistance mechanisms such as the chromosomal $\beta$ lactamases, $b l a_{\mathrm{OXA}-51-\text { like }}$ and $b l a_{\mathrm{ADC}}$, and increased activity of efflux pumps such as adeABC [24, 25]. A large 'resistance island' encoding more than a dozen antibiotic resistance genes was found in one of the first MDR $\mathrm{Ab}$ strains to be sequenced [26], and sequencing of hundreds of additional strains has shown that resistance genes are often associated with plasmids [27, 28]. The ISAba1 element and other insertion sequences have also contributed to antibiotic resistance by activating expression of intrinsic resistance genes adjacent to their insertion sites [29]. ISAba 1 elements are abundant in some Ab strains, and the location of ISAba1 insertions can add resolution to population dynamics and genomic epidemiology [30], but the role of these elements in adaptation to selective pressures during infections has not been addressed.

Clinicians have observed that in treating Ab infections, some patients remain chronically infected for prolonged periods of time. Whether long-term infected patients are the result of the persistence of one strain due to treatment failure, or new infection by a different strain, remains a pressing question. Genome sequencing provides an opportunity to address this question. We previously characterized the genetic profiles of $\mathrm{Ab}$ strains isolated from a large hospital system in the Midwestern USA and found considerable diversity including evidence for multiple closely related lineages and extensive variability of mobile elements [30]. In this followup study, we sought to understand what genome changes occur during the course of infection and antibiotic treatment. We therefore decided to sequence all available strains from patients that had multiple positive $\mathrm{Ab}$ cultures to characterize the nature of genetic change during infections. Longitudinal analysis of isolates collected during the course of infection and treatment can provide insight into infection history, and how Ab responds to host and antibiotic selective pressures.

\section{Methods}

\section{Strains}

A. baumannii strains identified in the University Hospitals Health System (UHHS) Clinical Microbiology Laboratory were stored in an archive since 2007. We identified all patients for which there were more than two isolates available in the archive as of 1 December 2013, and those isolates were selected for whole genome sequencing. A total of 136 new strains were sequenced and these genomes were combined for analysis with previously described sequences from UHHS [30] for a high resolution population-level analysis. Repeat cultures were ordered when patients presented with continued signs of infections. Clinical data, including treatment history and outcome, were collected from patient charts. The research study was reviewed and approved by the Institutional Review Board of the University Hospitals Case Medical Center, and conformed to the Helsinki Declaration.

\section{Sequencing}

Overnight cultures were grown in LB broth and DNA was isolated using an UltraClean ${ }^{\circ}$ Microbial DNA Isolation Kit (MoBio, Inc.) using a protocol implemented on an Eppendorf EpMotion automated pipetting device. Libraries were prepared for sequencing using Illumina NexteraXT kits and sequenced on an Illumina HiSeq 2000 with paired 100-base sequence reads. In general, more than 100-fold coverage was obtained for each genome. Each read set was assembled individually using velvet [31] and annotated using NCBI's PGAAP pipeline (http://www.ncbi.nlm.nih.gov/genome/annotation_prok/). All genomes are available at NCBI under BioProject PRJNA262565.

\section{Phylogenetic analysis}

A core phylogeny based on single nucleotide variants (SNVs) was inferred using SNVs identified by kSNP [32] and constructed using $R A x M L$ [33] with previously sequenced UHHS and other publicly available genomes included for phylogenetic context [30]. Genome positions with allele calls in at least $80 \%$ of strains were included in the analysis. Regions of the genome subject to recombination were excluded from consideration in construction of the core phylogeny as in Wright et al. [30].

\section{Variant detection analysis}

A more stringent approach was used to identify patientrestricted and isolate-specific SNVs from all patients with monophyletic isolates. Primary Illumina sequence reads were aligned to patient-specific reference genome assemblies, typically the earliest isolate from each patient, using $b w a$. To avoid the impact of low-quality sequences at contig edges, SNVs within 200 bases of contig edges were excluded. Variant-calling was performed using freebayes (arXiv:1207.3907 [q-bio.GN]) with a requirement for $\geq 20$ fold coverage of the alternative allele with no reads supporting the reference allele, and a minimum SNV quality of 500. SNVs identified when reads from the reference genome were aligned to the reference contigs were excluded as likely indicating errors in the reference assembly. Patient-specific SNVs were identified as those SNVs 
uniquely shared within a patient based on the kSNP output for the full set of SNVs annotated to the reference $\mathrm{Ab}$ ACICU genome. ACICU was the first sequenced MLST ST2 (Global Clone 2) isolate, obtained from Italy in 2005 [34], and was selected as a reference because the majority of isolates in this study are GC2. Synonymous/non-synonymous status was inferred based on alignment of flanking sequences to the $\mathrm{ACICU}$ annotation.

\section{Genome variation analysis}

To identify gene gain and loss events during infection, pan-genome analysis was performed using PanOCT [35]. The locations of IS elements in the draft genome assemblies was performed using ISSeeker, a custom perl script (https://github.com/JCVI-VIRIFX/ISseeker). IS locations in each genome were mapped to the common reference genome ACICU. The two most abundant IS elements in this population, ISAba1 and IS26 were examined and isolate-specific and patient-specific insertion sites were determined and mapped to the ACICU annotation.

\section{Functional enrichment analysis}

Functional roles and pathways were assigned using KEGG designations [36] and TIGRfams [37] for each nonsynonymous SNV and non-neutral IS event, and the number of genes within each category was determined for ACICU. To identify statistically significant enrichment in functional classes of genes, hypergeometric probability tests were conducted. In the calculation, the population size was the number of all genes in the PanOCT shared clusters for the 136 genomes (553,343 genes); the population successes value was calculated as the number of genes in each functional role or pathway category based on its abundance in ACICU multiplied by each genes' occurrence in the PanOCT shared clusters; sample size was the number of SNV or IS events for isolate-specific (IS), patient-restricted (PR), or patient-specific (PS) categories, respectively; and the number of sample successes was the number of hit genes in each category.

\section{Results}

\section{Isolate characteristics}

Genome sequences were obtained for $136 \mathrm{Ab}$ isolates from 40 patients (Additional file 1: Table S1 and Additional file 2: Table S2). The number of isolates per patient was in the range of 2-10, over 0-829 days between first and last isolation (Additional file 1: Table S1). The pattern of antimicrobial susceptibility indicated that 125/136 isolates were resistant to $\geq 3$ classes of antibiotics and are thus considered MDR. Isolates were collected from an integrated healthcare system: a central referral hospital (92/136), outlying community hospitals that were part of the healthcare system (23/136), long-term care facilities (16/136), and outpatient clinics (5/136). The isolates were collected from patients evaluated in an ICU, general medical wards, and from the emergency department. The 136 genomes were placed in the context of other UHHS genomes detailed in Wright et al. [30], and 22 publicly available reference genomes using a phylogeny constructed from 50,466 core SNVs located in non-recombinant regions of the genome (Fig. 1).

A patient was considered to have an infection stemming from one colonization event when all of the isolates from that patient were located on a monophyletic, well-supported terminal node of the core phylogeny. Twenty-five patients had such monophyletic infections (Fig. 2, Additional file 1: Table S1). Fifteen patients had polyphyletic infections with isolates located at clearly distinct branches of the phylogenetic tree, indicative of at least two independent colonization events. Two of these patients have co-occurring polyphyletic infections in which phylogenetically distinct strains were isolated in overlapping time intervals, while the other 13 polyphyletic patients were likely re-infected with new strain types. In some cases, multiple related (monophyletic) strains were isolated from patients that were also infected with genetically distinct strains (polyphyletic). There was no relationship between the number of days separating culture-positive isolations and the likelihood of whether a new isolate was closely related (monophyletic) or represented a reinfection (polyphyletic) (Additional file 1: Table S1). The site of infection also did not predict whether subsequent isolates would be related: there are examples of both monophyletic (for example, Patient 236) and polyphyletic infections (for example, Patient 348 ) occurring at different body sites in the same patient.

\section{Monophyletic isolates}

SNVs in monophyletic groups from individual patients were of particular interest and were examined in more detail. Three classes of SNVs were defined: isolate-specific (IS) SNVs were found only in a single isolate; patientrestricted (PR) SNVs were found in multiple isolates (but not all) from a single patient; and patient-specific (PS) SNVs were shared by all isolates from a patient. For all three classes, the SNVs were not observed in any other UHHS or public reference strain. The complete list of these SNVs with gene annotations is presented in Additional file 3: Table S3. Most of the SNVs that occurred during infection were unique to each isolate: of the 220 detected SNVs, 189 (86 \%) were isolate-specific. IS SNVs arising during infection were detected in 31 strains (mean 4.8 SNVs, range 0-29 SNVs/patient). PR SNVs were identified in nine patients (range: 0-8 SNVs/patient). There was no relationship between the number of SNVs per isolate and the number of days between isolations (Fig. 3). For example, the three isolates from Patient 236 collected on the same day had eight 


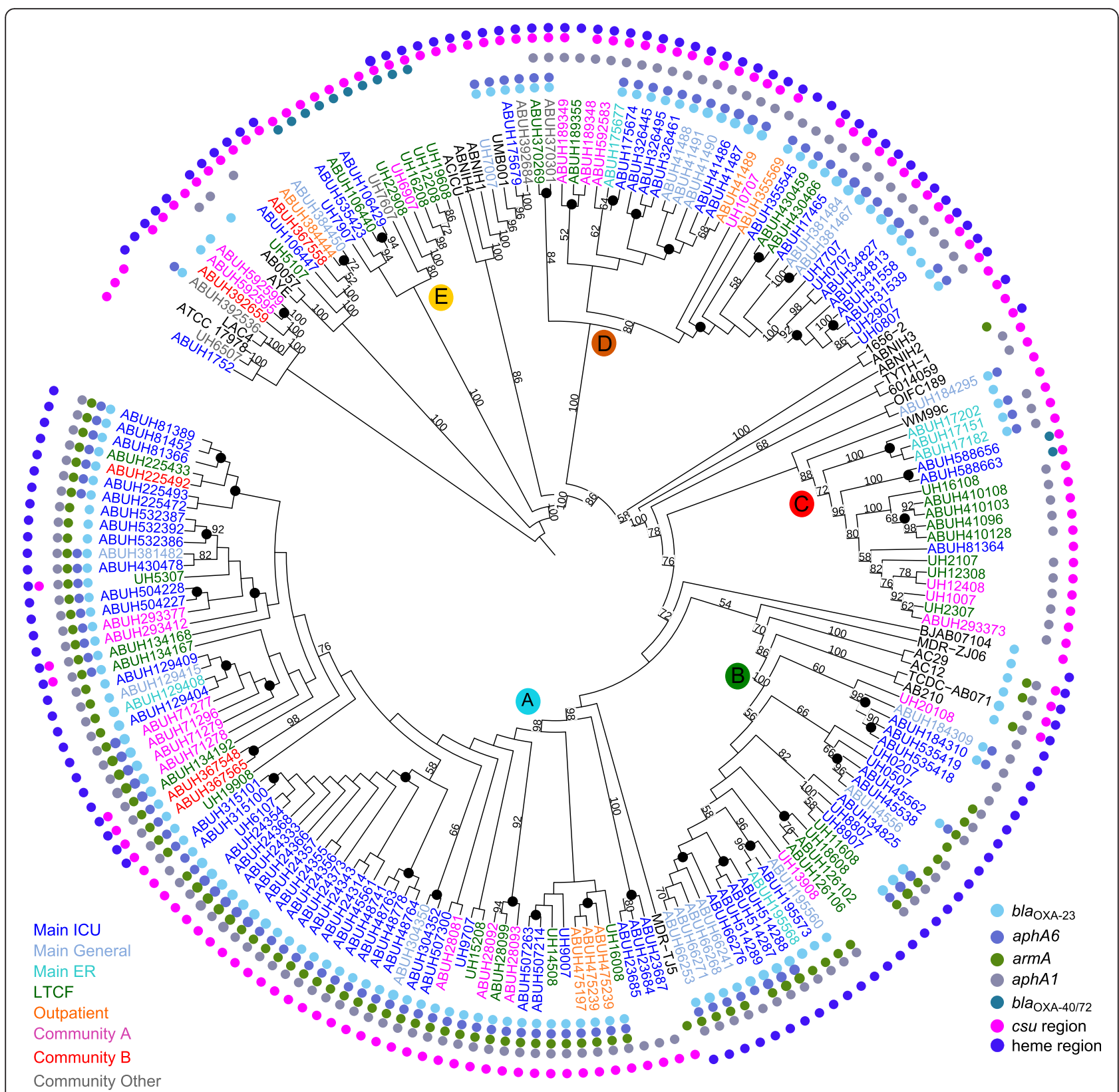

Fig. 1 Core SNV phylogeny of A. baumannii strains. A core phylogeny was constructed using 50,472 SNVs that were present in at least $80 \%$ of all strains. SNVs were identified using KSNP and the tree was built using RAXML. Clade A-E designation reflect primary clade structure previously designated $[30,44]$ where strains in Clade A-D through the ACICU branch are all Global Clone II, or multi-locus sequence type (MLST) ST2, strains [60]. Internal nodes with bootstrap support of at least $50 \%$ are indicated. Black circles near terminal nodes indicate highly-supported monophyletic groups of strains from individual patients. Strain names are color-coded based on the hospital from which the isolate was obtained and the first the numbers after ABUH refer to patient and strain identifiers in Additional file 1: Table S2. Colored dots represent the presence of specific genes or genomic regions of interest as listed in the key

SNVs among them, while three isolates from Patient 195 over 48 days had 0 detected SNVs among them. Isolates from distinct body sites did have more isolatespecific SNVs. For example, the two Clade B isolates from Patient 184 had 14 (percutaneous endoscopic gastrostomy site) and 15 (urinary tract infection) IS SNVs. SNVs that were restricted to a patient had a higher proportion of non-synonymous (NS) amino acid substitutions compared to synonymous (S) substitutions $(\mathrm{NS} /(\mathrm{NS}+\mathrm{S})$ : $0.97)$ than the isolate-specific (0.77) and patient-specific SNVs (0.74) (Table 1) yet all categories of isolate- and patient-restricted SNVs were strongly skewed towards non-synonymous mutations relative to the populationlevel NS/(NS + S) ratio of 0.18 . 


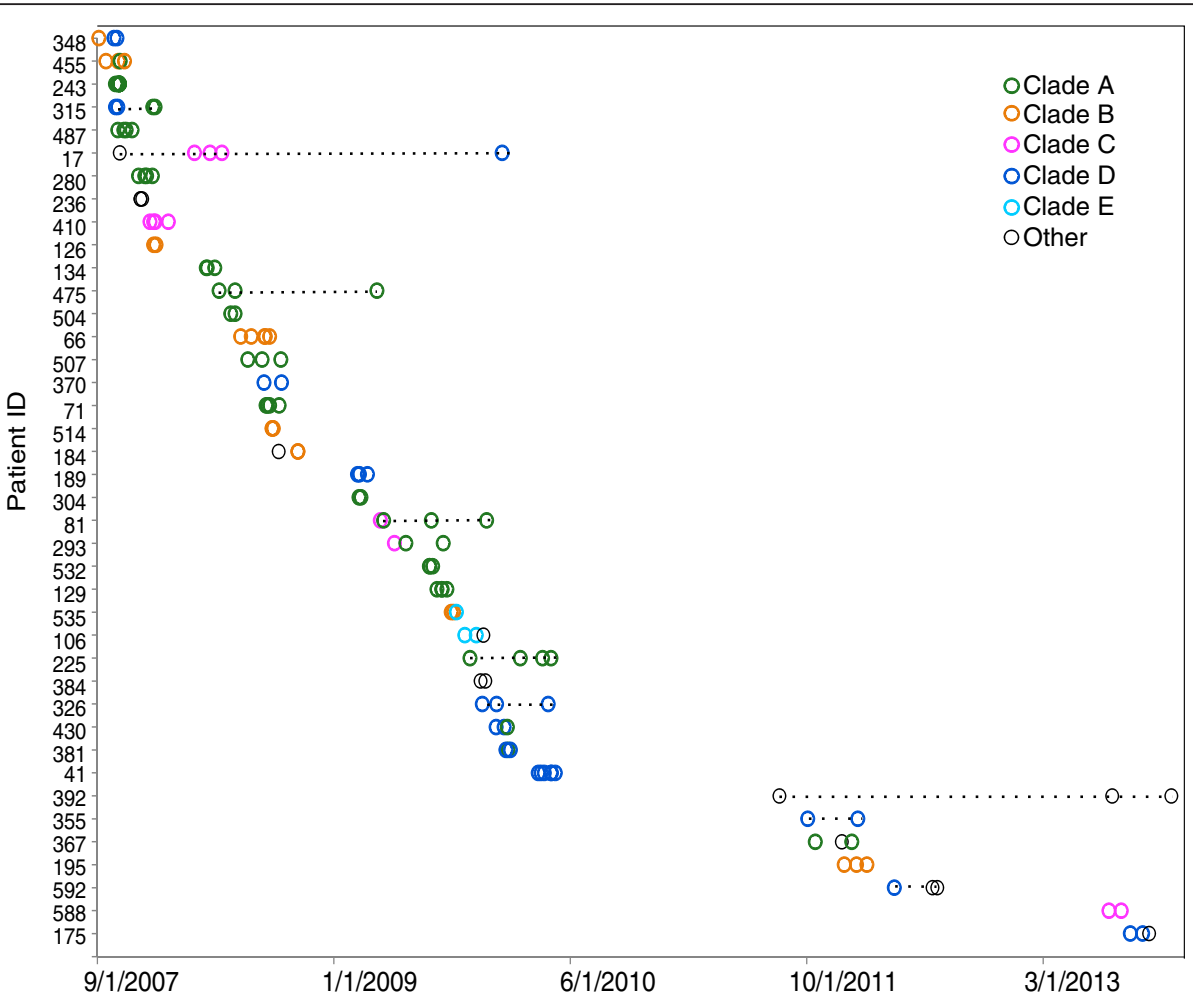

Fig. 2 Date of isolation for each longitudinal isolates for each patient. Clade designation was determined as described in Fig. 1

ISAba1 is abundant and dynamic in the UHHS population, and mapping of the insertion sites for ISAba1 revealed additional genomic modifications during infection. Similar to SNV patterns, most new ISAba1 insertions were isolatespecific and these tended to be in coding regions or in an

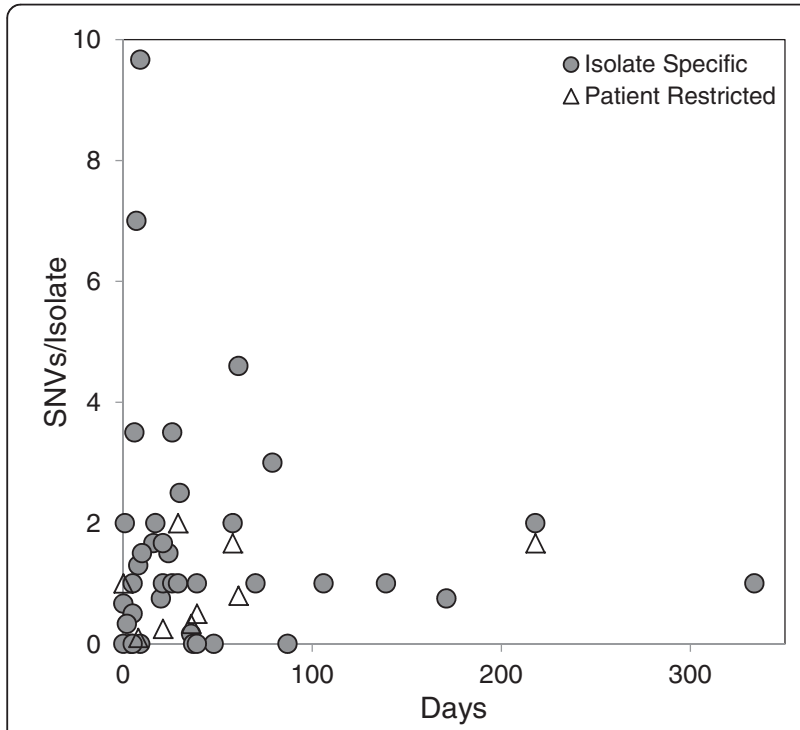

Fig. 3 Relationship between SNV frequency and time between strain isolations The number of within-patient SNVs is shown as a function of time for isolate-specific and patient-restricted SNVs for each patient orientation that would result in overexpression of an adjacent gene (Table 1, Additional file 4: Table S4). Only three patient-restricted IS events were detected, two of which are predicted to result in the overexpression of downstream genes. Independent ISAba1 insertions upstream of the fimA gene in two different patients were predicted to lead to overexpression of the type- 1 fimbrial protein. A smaller fraction of patient-specific IS events (uniquely shared among all isolates from a patient) were in coding sequences or an overexpression orientation, while across all UHHS isolates, ISAba1 was less likely to be found in an overexpression orientation.

The 10 strains isolated from Patient 243 provide particularly illustrative examples of SNV and ISAba1 patterns associated with temporal and spatial heterogeneity. Six isolates encompassing respiratory, urine, and abdominal sites of infection had no detectible SNVs among them, including three isolates cultured 8 days after the initial sample. The other four isolates had between two and six SNVs relative to Day 0 isolates. All but one these SNVs were isolate-specific, where the mutation in the RNA polymerase gene $r p o B$ was present in two Day 8 abdominal fluid isolates. No ISAba 1 insertions that arose during infection were shared among Patient 243 isolates. SNV patterns from Patient 236, on the other hand, reveal that spatial isolation can lead to strain differentiation. The three isolates are monophyletic, forming a terminal 
Table 1 SNV and IS event summary

\begin{tabular}{lllll}
\hline SNV & Intergenic $^{a}$ & Synonymous & Non-synonymous & NS/(N+S) \\
Isolate-specific & $30(16)$ & 36 & 123 & 0.77 \\
Patient-restricted & $2(7)$ & 1 & 28 & 0.97 \\
Patient-specific & $58(15)$ & 83 & 237 & 0.74 \\
Population-level $^{b}$ & $10,709(9)$ & 85,865 & Neutral & 0.18 \\
ISAba| $^{c}$ & Overexpressed & Loss of function & $3(13)$ & Total \\
Isolate-specific $_{\text {Patient-restricted }}$ & $8(33)$ & $13(54)$ & $1(33)$ & 3 \\
Patient specific & $2(67)$ & 0 & $7(28)$ & 24 \\
Population-level & $8(32)$ & $10(40)$ & $21(25)$ & 84 \\
\hline
\end{tabular}

${ }^{a}$ Values in parentheses are the percentage of SNVs that are intergenic

${ }^{\text {bS }}$ ummary from kSNP output analyzing the 136 longitudinal isolates, and UHHS and public genomes

'Values in parentheses are the percentage of IS events in each category

branch with distinct SNVs and gene content from other UHHS strains. The blood and abdominal fluid isolates (ABUH23684 and ABUH23685) have three shared SNVs and one ISAba 1 insertion not present in ABUH23687 (a scrotal isolate) which has two unique SNVs.

Gene content changes in monophyletic isolates were primarily gene loss events centered around IS26-mediated deletions in the astA and ACICU_02399 Tn1548 resistance island regions described in [30]. IS26 has been shown to cause deletions of adjacent sequence [38, 39]. We observed a partial deletion of $\operatorname{Tn} 1548$ at the catB gene in ABUH315100 which resulted in the loss of the amikacin resistance gene, $\operatorname{arm} A$, and independent losses of the adjacent $c s u E$ region in multiple isolates as best depicted by the variable presence of $c s u E$ in isolates from clades B and D (Fig. 1). Variation in phage content was observed in Patient 410 in a region previously described as dynamic [30]. The first Patient 410 isolate had one $48 \mathrm{kbp}$ region of phage sequence, the second had an additional $36 \mathrm{kbp}$ of sequence, and the last two isolates had an additional $13 \mathrm{kbp}$ of sequence in this region, resulting in a variable phage region in the range of 48-97 kbp in size.

\section{Polyphyletic isolates}

Two patients had co-occurring polyphyletic infections in the same infection site: bronchial specimens from Patient 367 and Patient 455 produced isolates from different clades. There was no evidence for genetic exchange between the Clade A strains in Patient 367 and the more divergent ABUH367558, which was isolated at an intermediate time point between the two clade A strains. The two clade A strains are isogenic, as they share a terminal branch on the tree, with only three SNVs that distinguish them and a uniquely shared ISAba1 insertion in a gene encoding the histidine kinase protein of an uncharacterized two component regulatory system (TCRS) (ACICU_03157). This strain persisted largely unchanged during the 77 days between isolations. Genetic exchange did occur between the two different strain types in Patient 455: pABUH1 was transferred from the clade A strain to the clade B strain (ABUH45561 to ABUH45538/ABUH45562) as previously described [30].

Thirteen patients had polyphyletic infections where reinfection or replacement with a new strain type was likely. Clade A strains carry the most resistance determinants and were not replaced with a different strain type in this population.

\section{Enrichment and convergence}

Analysis of the functional categories of genes with SNVs and IS insertions demonstrated that genes associated with antibiotic resistance and host interaction are overrepresented (Table 2 ). The most commonly mutated genes were $\operatorname{pmr} A B$ that encode the two component regulatory system (TCRS) involved in mediating colistin resistance through modification of surface polysaccharides and membrane charge [40-43]. Twelve patients had at least one isolate with a mutation in $p m r A$ or $p m r B$ and four of those patients had multiple strains with independent mutations (Fig. 4a). The adeRS genes, which encode a TCRS that regulates expression of the efflux pump operon ade $A B C$, were the second most commonly mutated loci, found in isolates from 12 patients (Fig. 4b). Seven patients had mutations in both the pmrAB and adeRS TCRS genes. Another uncharacterized histidine kinase component of a TCRS, ACICU_03157, had both ISAba1 and point mutations in three patients. Transporters were also significantly enriched, accounting for 30 out of 123 non-synonymous SNVs in the isolate-specific SNV set. This included multiple cation (copA, znuB (two independent mutations) and $\operatorname{ars} B$ ) and iron-related transporters (fhuE (two independent mutations), $f e o B$, and siderophore transporters), as wells as antibiotic transporters including the porin carO, adeB, and adeJ efflux pumps, and those associated with lipopolysaccharide export, $m s b A$ and $l p t F$. The iron 
Table 2 Enrichment analysis of genes with SNV and IS events. Significant enrichment in classes and pathways assessed using hypergeometric distribution test based on abundance of genes in each category in ACICU, integrated with PanOCT shared clusters report (see text for detail)

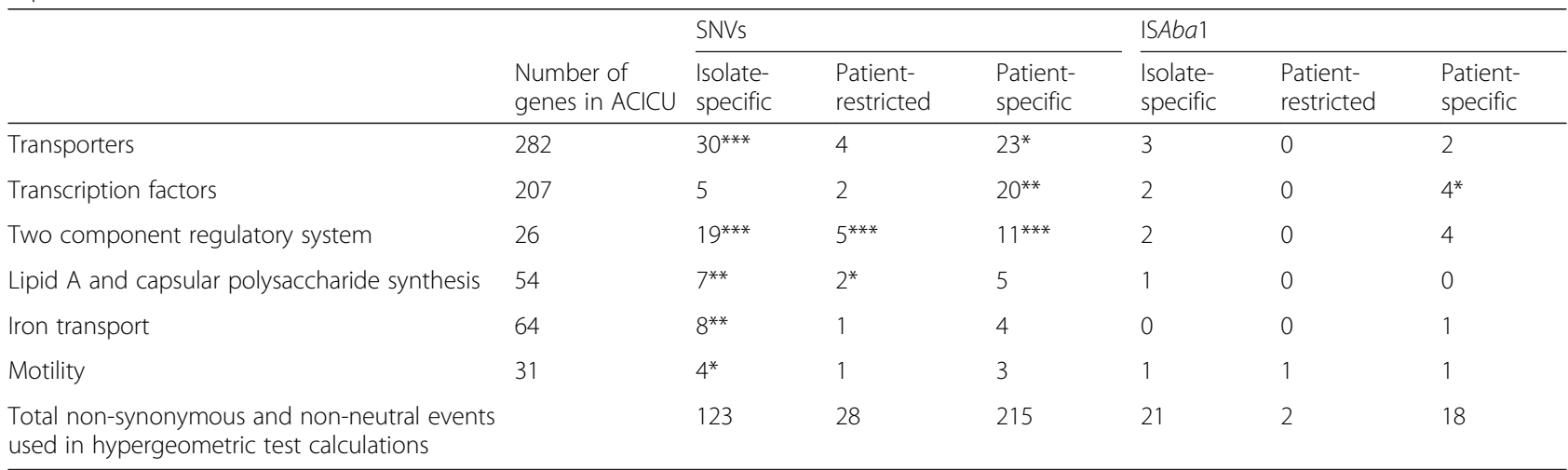

$*<0.05, * *<0.005, * * *<0.0005$

acquisition gene, $i u c D$, had both NS SNV and IS mutations in strains from four patients (Fig. 3c). Motility and adhesion related genes were also significantly enriched (for example, csuE, pilG, and fimA). Mutations in the tyrosine kinase $w c z$ gene involved in capsular polysaccharide assembly were detected in three patients, while UDP-N-acetylmuramate-alanine ligase murD mutations were observed twice. The RNA polymerase III gene, $r p o B$, had three NS mutations in two patients; all of the SNVs were located in the rifampin resistance-determining region (RRDR).

ISAba1 insertion sites also tended to be in genes with functions in antibiotic resistance and host adaptation. Genes encoding transporters (including AdeABC efflux pump), transcriptional regulators, surface-associated genes, and secreted and adhesion genes like $p a p D$ and $\operatorname{fim} A$, were all modified by ISAba1 (Additional file 4: Table S4).

\section{New insights into the population structure of}

\section{A. baumannii population at $\mathrm{UH}$}

The primary clade structure described previously is unchanged with the addition of the strains analyzed here [30, 44]. Clade $\mathrm{A}$ is most abundant, with 58 isolates. The analysis of the expanded set of UHHS strains revealed that there are two clade A subgroups that differ in the presence of a region that encodes a heme oxygenase, hem $\mathrm{O}$, (Fig. 1) that was previously described as a recombination hotspot $[30,45,46]$. There is evidence for more divergent strains among the most recent isolates that carry new genetic material, including the $b l a_{\mathrm{OXA}-72}$ variant of the $b l a_{\mathrm{OXA} 24 / 40}$-type carbapenemase gene in ABUH588656 and ABUH588663. There is now evidence for recombination at the $b l a_{\text {OXA-51 }}$ locus in clade $\mathrm{C}$, with a different allele now present.

Epidemiological and genomic data highlight multiple cases of likely patient-to-patient transmission. For example, the clade A cluster of isolates from Patients 243, 315 , and 487 all occurred in the ICU within the span of a few days in 2007. These three patients share four unique
SNVs, while Patients 243 and 487 share an ISAba1 event. Similarly, Patient 66 and Patient 514 overlapped in the ICU in 2008 and had nearly identical clade B isolates with one shared SNV (1 bp upstream from start of ACICU_ 01396, a predicted MFS transporter).

Patients with persistent infections move among hospital locations during the course of treatment. Eleven patients contributed isolates obtained in more than one hospital, with transfer between a long-term care facility (LTCF) and the tertiary care hospital being the most common transfer route (four patients moved to LTCF, four patients were transferred from a LTCF to the main or regional hospitals). Patient 392 was infected with three different strains over the course of $>800$ days, each isolated during a stay at a different community hospital. Patient 225 was infected with a monophyletic set of strains from clade A over 170 days, with strains isolated during the patient's stay at a LTCF, the main ICU ward, a community hospital, and again at the main ICU ward.

\section{Discussion}

Two lines of evidence suggest that natural selection is contributing to the range of SNVs and IS insertions observed in the series of longitudinal isolates from each patient. First, genes involved in antibiotic resistance and host interaction were significantly enriched for novel genetic variants. MDR strains have limited treatment options, resulting in an increased reliance on last-line therapies like colistin and tigecylcine. The $p m r A B$ genes were the most frequently mutated, with 18 independent mutations and IS events observed. These mutations tended to occur in patients that had been treated with colistin: 12 patients had isolates with mutations in these loci, and at least nine of them received colistin therapy. Colistin treatment status was unknown for the other three patients due to incomplete patient histories. Mutations in pmrAB that arise during treatment have been described previously [47]. Ample evidence supports a role 


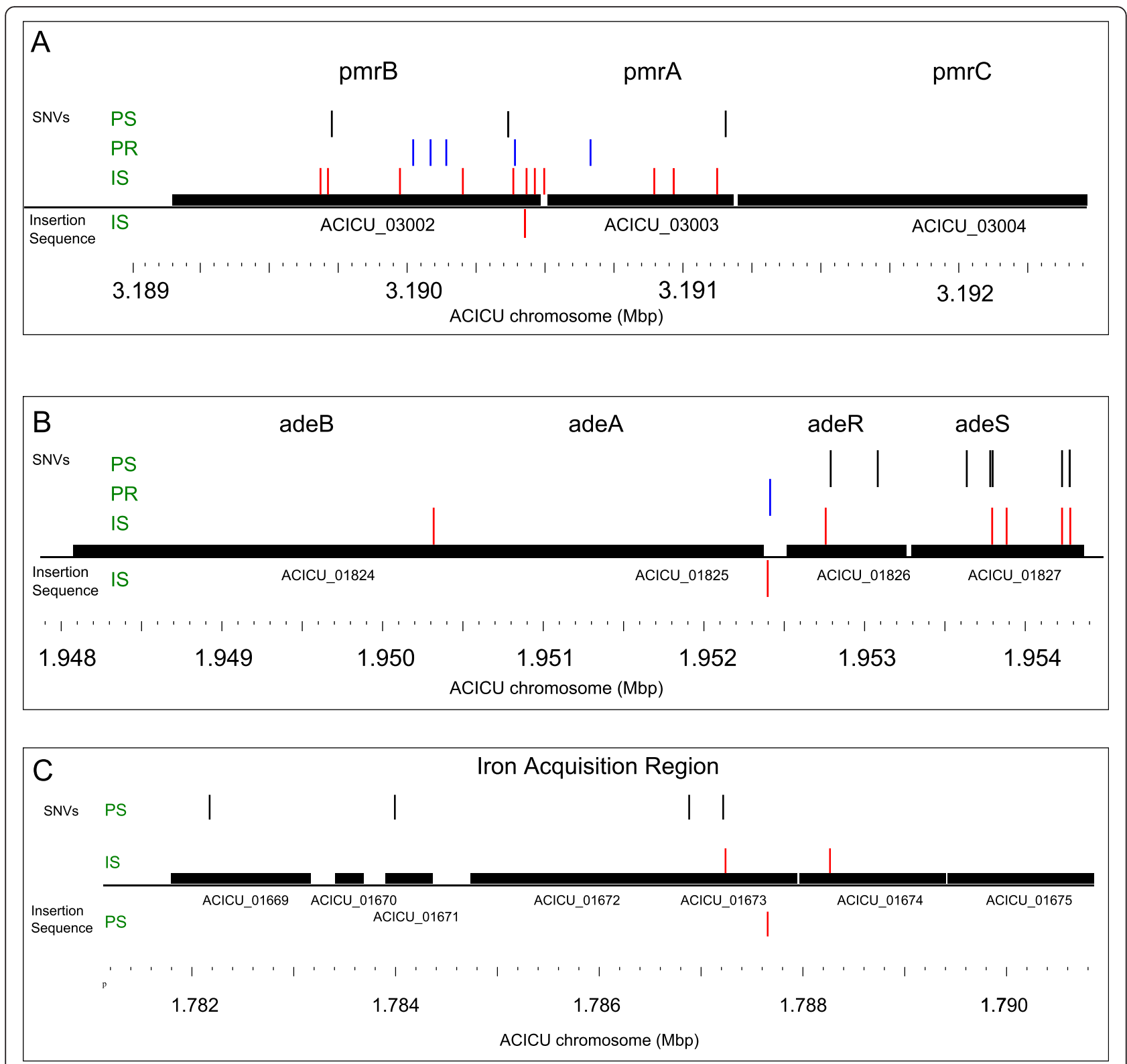

IS: Isolate Specific

PR: Patient Restricted PS: Patient Specific

Fig. 4 Selected loci with multiple independent mutations. Genes and gene regions that exhibited a significantly over-representation of mutation frequency are shown. a The pmrCAB genes encode the PmrAB TCRS that is associated with resistance to colistin and one of the target genes (pmrC), that encodes a lipid A phosphoethanolamine transferase. $\mathbf{b}$ The adeABC genes encode an RND family efflux pump that is involved in resistance to several classes of antibiotics. The adeRS genes encode a TCRS that regulates adeABC. $\mathbf{c}$ A region encoding several genes involved in siderophore biosynthesis. Iron acquisition mediated by siderophores is an important adaptive mechanism in the host environment that is characterized by limited free iron concentrations

for the TCRS system PmrAB in regulation of colistin resistance in $\mathrm{Ab}$ through upregulation of $\mathrm{pmrC}$, a lipid A phosphoethanolamine transferase [41, 42]. Some relationships between $\operatorname{pmr} A B$ mutation and colistin minimum inhibitory concentration (MIC) were observed. For example, the MIC for isolate ABUH41489 with an ISAba125 interruption of $p m r B$ was $>128 \mathrm{ug} / \mathrm{mL}$, while isolate ABUH41488 from the same patient, which had a $p m r A$ mutation (I173F) had a MIC measured at $2 \mathrm{ug} / \mathrm{mL}$. However, the majority of strains with $p m r$ mutations had MIC values 
$<2 \mu \mathrm{g} / \mathrm{mL}$, which is considered phenotypically susceptible in standard clinical assays (Additional file 2: Table S2). A recent study of the development of resistance in the context of colistin therapy in A. baumannii showed that clinical colistin resistance can go undetected by standard clinical methodologies [48]. Though phenotypically susceptible, $p m r C$ expression values were significantly increased from 5-30-fold in three strains tested relative to their isogenic parental strains from the same patient (RNASeq data not shown). Thus the elevated $p m r C$ expression suggests that pmrAB mutations in these strains may result in lipid A modifications that confer some level of protection from colistin. Mutations in $\operatorname{lp} x A C D$, encoding for lipid A biosynthesis pathway, have also been implicated in conferring colistin resistance [49]. We did not observe any mutations in the lpx genes, supporting the prediction that $p m r A B$ mutations are likely to be the most commonly encountered colistin resistance mechanism in $\mathrm{Ab}$ in clinical settings due to a higher fitness cost of $l p x A C D$ mutations [50].

Another TCRS, adeRS, and its associated efflux system, ade $A B C$, also had multiple independent mutations in this population, with 16 independent mutations and IS events in 12 patients. AdeRS regulates the expression of the ade $A B C$ efflux pump genes which are associated with resistance to several antibiotics including to tigecycline [51, 52]. Sequence variation is high in these genes as observed previously and related to tigecyline resistance [53]. Interestingly, only three patients with adeRS or adeABC mutations in this study were confirmed to have received tigecycline during treatment, while four did not, and five patients had inconclusive patient records. All adeRS and adeABC mutant isolates had tigecycline MIC values $>2 \mu \mathrm{g} / \mathrm{mL}$ (Additional file 2: Table S2), indicative of a resistant phenotype. Eight of the 14 mutations were patient-specific, meaning that even the initial isolate had the mutation. This observation is consistent with the hypothesis that the regulation of ade $A B C$ is under selection for more than just tigecycline efflux [51]. Mutations in the $r p o B$ gene encoding RNA polymerase III can confer resistance to rifampin. Three isolates from two patients had $r p o B$ mutations in the rifampin resistance determining region (RRDR) [54] and both patients received rifampin therapy in combination with colistin. This suggests that mutations in other multiply-mutated genes may also have adaptive value in the context of infection.

MDR infections still have to persist in the context of the host immune system, and functional categories related to regulation of transcription, membrane transport, surface polysaccharide synthesis and organization, iron acquisition, and motility were enriched in mutations. Mutations were observed in the tyrosine kinase $w z c$ gene involved in mediating capsular polysaccharide organization [55-57]. Notably, several mutations occurred in genes encoding iron acquisition and siderophores with potential implications for iron acquisition during host infection. The enrichment in transcription factors in SNV and IS events raises the question of how the transcriptional landscape changes during infection. For example, the histidine kinase component of an uncharacterized TCRS, ACICU_03157, had both SNVs and IS mediated changes in this population.

The second line of evidence of a role for natural selection in the set of observed SNVs is the elevated ratio of non-synonymous variants compared to the population-wide average (Table 2). Isolate-and patientspecific SNVs were much more likely to be nonsynonymous than SNVs observed in isolates from multiple patients. SNVs in multiply-hit genes also have higher nonsynonymous ratios at the population level compared to the population mean $((\mathrm{NS}) /(\mathrm{NS}+\mathrm{S})=0.18)$ (Additional file 5: Table S5), suggesting that these genes may be subject to diversifying selection, in contrast to those genes below the population-wide value. For example the iron-related genes, ACICU_01673, ACICU_01696, ACICU_02572, and ACICU_02581, all have populationwide $((\mathrm{NS}) /(\mathrm{NS}+\mathrm{S})>0.35$. Furthermore, siderophore synthesis genes, transporters, and TCRS, were also observed in strain-specific SNVs in an Ab outbreak [58]. Functional gene enrichment findings are also in agreement with other longitudinal studies including persistent and asymptomatic Burkholderia pseudomallei infection which had key changes in lipopolysaccharide biosynthesis and modification [10], and those observed during 12 weeks of Staphylococcus aureus blood stream infection that included transporters and antibiotic resistance genes [4]. Lieberman et al. (2014) found extensive diversity of Burkholderia dolosa in individual CF patients, with multiple adaptive mutations appearing, but few becoming fixed in the population over time [6]. In a longitudinal study of over 400 Pseudomonas aeruginosa strains isolated from 34 CF patients, convergent evolution was identified in several genes involved in host adaptation based on remodeling of regulatory networks [59]. An alternative explanation, however, for the elevated rate of nonsynonymous mutations is that there has not been adequate time for deleterious mutations to be removed from the population. This alternative hypothesis predicts that over time the fraction of non-synonymous mutations would decline. More extensive sampling of infected patients will be required to better understand the spectrum of mutations that arise during infection and make inferences about their functional effect.

Hospital-acquired infections are generally considered acute and treatable. Increasing rates of antibiotic resistance are changing that perception. Strains resistant to all FDAapproved drugs now represent a significant fraction of Ab infections in many hospitals and have led to long-term infections that are difficult to treat. Relatively few studies have examined the evolution of bacterial pathogens during 
long-term infections. We found that patients persistently infected with MDR Ab are due to both treatment failure and re-infection with new strains. Multiple examples were found of patients infected with the same strain for $>150$ days. Patient movement data highlight the need to monitor patients with a history of MDR Ab as they are admitted to a new hospital unit.

A limitation of this study is that serial sampling of infected patients was not performed in a systematic or prospective manner, but relied on a convenience sample that may introduce biases. Another limitation is that a single isolate was sequenced from each culture date and so we were not able to evaluate the population of variants that were present in a patient at any given time. In some cases the retrospective sampling design does not distinguish between co-occurring, replacement, or re-infection scenarios. To more rigorously distinguish between these classifications, multiple isolates from multiple time points need to be analyzed. Nonetheless, we were able to show that both long-term infections and super-infections with genetically distinct strains are common.

\section{Conclusions}

Genomic analysis of A. baumannii isolates collected from the same patient over time revealed enrichment in mutations associated with antibiotic and host responses, including those encoding for transcriptional regulators, cell surface structures, and iron utilization. Most mutations were isolate-specific, yet enrichment analysis suggests that the much of the genetic variation detected is under selection.

\section{Additional files}

Additional file 1: Table S1. Summary of patient and isolate data. Clade designation and infection type (monoclonal, polyclonal, mixed) is based on strain relatedness in the core phylogeny (Fig. 1). Number of SNVs (isolate-specific, patient-restricted, patient-dependent); number of ISAbaI events (isolate-specific, patient-restricted, patient-dependent). (XLSX 32 kb)

Additional file 2: Table S2. Isolate metadata including date and location of isolation, and antibiotic susceptibility data. Colistin susceptibility was assayed using both E-test strips (bioMérieux) and broth microdilution tests (Sensititre platform). (XLSX $63 \mathrm{~kb}$ )

Additional file 3: Table S3. Isolate-specific, patient-restricted, patientspecific SNVs. (XLSX $64 \mathrm{~kb}$ )

Additional file 4: Table S4. Isolate-specific, patient-restricted, patientspecific ISAbal events. (XLSX $17 \mathrm{~kb}$ )

Additional file 5: Table S5. Multiply mutated genes. All genes with multiple SNV and ISAbaI events are listed with their predicted function. The text color indicates what the class of mutations are: SNV or ISAbaI events, and whether the mutation was isolate-specific, patient-restricted, or patient-dependent. Green: IS SNV; blue: PR SNV; purple: PS SNV; orange: IS ISAba1; brown: PR ISAba1; pink: PS ISAba1. (XLSX 20 kb)

\section{Authors' contributions}

MW analyzed the genomic data and drafted the manuscript. Al collected and summarized patient treatment history. MJ carried out isolate phenotypic characterization. RB coordinated clinical aspects and participated in the design of the study. MA designed the study and drafted the manuscript. All authors read and approved the final manuscript.

\section{Acknowledgements}

We thank the JCVI Sequencing Core Facility and Informatics team, as well as Saralee Bajaksouzian for their contributions to this project. MDA is supported by a grant from the National Institute of General Medical Sciences of the National Institutes of Health (NIH) through award number R01GM094403. RAB is supported by the Department of Veterans Affairs VISN 10 Geriatrics Research, Education and Clinical Center, by the Department of Veterans Affairs Office of Research and Development under Award Number I01BX001974, by the National Institute of Allergy and Infectious Diseases (NIAID) of the National Institutes of Health (NIH) under Award Numbers UM1Al104681, R01Al072219, R01Al063517 and R01Al100560. Additionally, RAB has received grants from AstraZeneca, Merck, and Melinta. The content is solely the responsibility of the authors and does not represent the official views of the Department of Veterans Affairs or the National Institutes of Health. The funders had no role in study design, data collection and interpretation, or the decision to submit the work for publication.

\section{Author details}

${ }^{1}$ The J. Craig Venter Institute, La Jolla, CA, USA. ${ }^{2}$ Department of Medicine, University Hospitals Case Medical Center, Cleveland, OH, USA. ${ }^{3}$ Department of Pathology, University Hospitals Case Medical Center, Cleveland, OH, USA. ${ }^{4}$ Department of Pathology, Case Western Reserve University, Cleveland, $\mathrm{OH}$ USA. ${ }^{5}$ Departments of Pharmacology, Molecular Biology and Microbiology, and the Center for Proteomics, Case Western Reserve University, Cleveland, $\mathrm{OH}$, USA. 'Louis Stokes Cleveland Department of Veterans Affairs Medical Center, Cleveland, $\mathrm{OH}$, USA.

Received: 19 October 2015 Accepted: 10 February 2016

Published online: 03 March 2016

\section{References}

1. Dijkshoorn L, Nemec A, Seifert H. An increasing threat in hospitals: multidrugresistant Acinetobacter baumannii. Nat Rev Microbiol. 2007:5(12):939-51.

2. Fitzpatrick MA, Ozer E, Bolon MK, Hauser AR. Influence of ACB complex genospecies on clinical outcomes in a U.S. hospital with high rates of multidrug resistance. J Infect. 2015;70(2):144-52.

3. Kennemann $\mathrm{L}$, Didelot $\mathrm{X}$, Aebischer $\mathrm{T}$, Kuhn $\mathrm{S}$, Drescher B, Droege M, et al. Helicobacter pylori genome evolution during human infection. Proc Natl Acad Sci U S A. 2011;108(12):5033-8.

4. Mwangi MM, Wu SW, Zhou Y, Sieradzki K, de Lencastre H, Richardson P, et al. Tracking the in vivo evolution of multidrug resistance in Staphylococcus aureus by whole-genome sequencing. Proc Natl Acad Sci U S A. 2007;104(22):9451-6.

5. Wen H, Wang K, Liu Y, Tay M, Lauro FM, Huang H, et al. Population dynamics of an Acinetobacter baumannii clonal complex during colonization of patients. J Clin Microbiol. 2014:52(9):3200-8.

6. Lieberman TD, Flett KB, Yelin I, Martin TR, McAdam AJ, Priebe GP, et al. Genetic variation of a bacterial pathogen within individuals with cystic fibrosis provides a record of selective pressures. Nat Genet. 2014;46(1):82-7.

7. Lieberman TD, Michel JB, Aingaran M, Potter-Bynoe G, Roux D, Davis Jr MR, et al. Parallel bacterial evolution within multiple patients identifies candidate pathogenicity genes. Nat Genet. 2011;43(12):1275-80.

8. Markussen T, Marvig RL, Gomez-Lozano M, Aanaes K, Burleigh AE, Hoiby N, et al. Environmental heterogeneity drives within-host diversification and evolution of Pseudomonas aeruginosa. MBio. 2014;5(5):e01592-01514.

9. Marvig RL, Johansen HK, Molin S, Jelsbak L. Genome analysis of a transmissible lineage of Pseudomonas aeruginosa reveals pathoadaptive mutations and distinct evolutionary paths of hypermutators. PLoS Genet. 2013;9(9):e1003741.

10. Price EP, Sarovich DS, Mayo M, Tuanyok A, Drees KP, Kaestli M, et al. Within-host evolution of Burkholderia pseudomallei over a twelve-year chronic carriage infection. MBio. 2013;4(4):e00388-13. 
11. Perez F, Hujer AM, Hujer KM, Decker BK, Rather PN, Bonomo RA. Global challenge of multidrug-resistant Acinetobacter baumannii. Antimicrob Agents Chemother. 2007;51(10):3471-84.

12. Maragakis LL, Perl TM. Acinetobacter baumannii: epidemiology, antimicrobial resistance, and treatment options. Clin Infect Dis. 2008;46(8):1254-63.

13. McGowan Jr JE. Resistance in nonfermenting gram-negative bacteria: multidrug resistance to the maximum. Am J Med. 2006;119(6 Suppl 1):S29-36. discussion S62-70.

14. Paterson DL. The epidemiological profile of infections with multidrugresistant Pseudomonas aeruginosa and Acinetobacter species. Clin Infect Dis. 2006;43 Suppl 2:S43-48.

15. Sunenshine RH, Wright MO, Maragakis LL, Harris AD, Song X, Hebden J, et al. Multidrug-resistant Acinetobacter infection mortality rate and length of hospitalization. Emerg Infect Dis. 2007;13(1):97-103.

16. Perez F, Endimiani A, Ray AJ, Decker BK, Wallace CJ, Hujer KM, et al. Carbapenem-resistant Acinetobacter baumannii and Klebsiella pneumoniae across a hospital system: impact of post-acute care facilities on dissemination. J Antimicrob Chemother. 2010;65(8):1807-18.

17. Doi Y, Husain S, Potoski BA, McCurry KR, Paterson DL. Extensively drug-resistant Acinetobacter baumannii. Emerg Infect Dis. 2009;15(6):980-2.

18. Falagas ME, Rafailidis PI, Matthaiou DK, Virtzili S, Nikita D, Michalopoulos A. Pandrug-resistant Klebsiella pneumoniae, Pseudomonas aeruginosa and Acinetobacter baumannii infections: characteristics and outcome in a series of 28 patients. Int J Antimicrob Agents. 2008;32(5):450-4

19. Lin MF, Yang CM, Lin CH, Huang ML, Tu CC, Liou ML. Clinical features and molecular epidemiology of multidrug-resistant Acinetobacter calcoaceticus-A baumannii complex in a regional teaching hospital in Taiwan. Am J Infect Control. 2009;37(9):e1-3.

20. Lautenbach E, Synnestvedt M, Weiner MG, Bilker WB, Vo L, Schein J, et al. Epidemiology and impact of imipenem resistance in Acinetobacter baumannii. Infect Control Hosp Epidemiol. 2009;30(12):1186-92.

21. Park YK, Peck KR, Cheong HS, Chung DR, Song JH, Ko KS. Extreme drug resistance in Acinetobacter baumannii infections in intensive care units, South Korea. Emerg Infect Dis. 2009;15(8):1325-7.

22. Metan G, Sariguzel F, Sumerkan B. Factors influencing survival in patients with multi-drug-resistant Acinetobacter bacteraemia. Eur J Intern Med. 2009;20(5):540-4.

23. Gordon NC, Wareham DW. A review of clinical and microbiological outcomes following treatment of infections involving multidrug-resistant Acinetobacter baumannii with tigecycline. J Antimicrob Chemother. 2009;63(4):775-80.

24. Sun JR, Perng CL, Chan MC, Morita Y, Lin JC, Su CM, et al. A truncated AdeS kinase protein generated by ISAba1 insertion correlates with tigecycline resistance in Acinetobacter baumannii. PLoS One. 2012:7(11):e49534.

25. Yoon EJ, Chabane YN, Goussard S, Snesrud E, Courvalin P, De E, et al. Contribution of resistance-nodulation-cell division efflux systems to antibiotic resistance and biofilm formation in Acinetobacter baumannii. MBio. 2015:6(2):e00309-15.

26. Vallenet D, Nordmann P, Barbe V, Poirel L, Mangenot S, Bataille E, et al. Comparative analysis of Acinetobacters: three genomes for three lifestyles. PLoS One. 2008;3(3):e1805.

27. Mosqueda N, Gato E, Roca I, Lopez M, de Alegria CR, Fernandez Cuenca F, et al. Characterization of plasmids carrying the blaOXA-24/40 carbapenemase gene and the genes encoding the AbkA/AbkB proteins of a toxin/antitoxin system. J Antimicrob Chemother. 2014;69(10):2629-33.

28. Nigro SJ, Holt KE, Pickard D, Hall RM. Carbapenem and amikacin resistance on a large conjugative Acinetobacter baumannii plasmid. J Antimicrob Chemother. 2015;70(4):1259-61.

29. Turton JF, Ward ME, Woodford N, Kaufmann ME, Pike R, Livermore DM, et al. The role of ISAba1 in expression of OXA carbapenemase genes in Acinetobacter baumannii. FEMS Microbiol Lett. 2006;258(1):72-7.

30. Wright MS, Haft DH, Harkins DM, Perez F, Hujer KM, Bajaksouzian S, et al. New insights into dissemination and variation of the health care-associated pathogen Acinetobacter baumannii from genomic analysis. MBio. 2014;5(1):e00963-00913.

31. Zerbino DR. Using the Velvet de novo assembler for short-read sequencing technologies. Curr Protoc Bioinformatics. 2010;31:11.5:11.5.1-11.5.12.

32. Gardner SN, Hall BG. When whole-genome alignments just won't work: KSNP V2 software for alignment-free SNP discovery and phylogenetics of hundreds of microbial genomes. PLoS One. 2013;8(12):e81760.
33. Stamatakis A, Ludwig T, Meier H. RAxML-III: a fast program for maximum likelihood-based inference of large phylogenetic trees. Bioinformatics. 2005;21(4):456-63

34. lacono $M$, Villa $L$, Fortini $D$, Bordoni $R$, Imperi $F$, Bonnal $R$, et al. Wholegenome pyrosequencing of an epidemic multidrug-resistant Acinetobacter baumannii strain belonging to the European clone II group. Antimicrob Agents Chemother. 2008;52(7):2616-25.

35. Fouts DE, Brinkac L, Beck E, Inman J, Sutton G. PanOCT: automated clustering of orthologs using conserved gene neighborhood for pangenomic analysis of bacterial strains and closely related species. Nucleic Acids Res. 2012;40(22):e172.

36. Kanehisa M, Goto S, Sato Y, Kawashima M, Furumichi M, Tanabe M. Data, information, knowledge and principle: back to metabolism in KEGG. Nucleic Acids Res. 2014;42(Database issue):D199-205.

37. Haft DH, Selengut JD, Richter RA, Harkins D, Basu MK, Beck E. TIGRFAMs and Genome Properties in 2013. Nucleic Acids Res. 2013:41(Database issue):D387-395.

38. Dawes FE, Kuzevski A, Bettelheim KA, Hornitzky MA, Djordjevic SP, Walker MJ. Distribution of class 1 integrons with IS26-mediated deletions in their 3'-conserved segments in Escherichia coli of human and animal origin. PLoS One. 2010;5(9):e12754.

39. He S, Hickman AB, Varani AM, Siguier P, Chandler M, Dekker JP, et al. Insertion sequence IS26 reorganizes plasmids in clinically isolated multidrug-resistant bacteria by replicative transposition. MBio. 2015;6(3):e00762.

40. Adams MD, Nickel GC, Bajaksouzian S, Lavender H, Murthy AR, Jacobs MR, et al. Resistance to colistin in Acinetobacter baumannii associated with mutations in the PmrAB two-component system. Antimicrob Agents Chemother. 2009;53(9):3628-34.

41. Arroyo LA, Herrera CM, Fernandez L, Hankins JV, Trent MS, Hancock RE. The pmrCAB operon mediates polymyxin resistance in Acinetobacter baumannii ATCC 17978 and clinical isolates through phosphoethanolamine modification of lipid A. Antimicrob Agents Chemother. 2011;55(8):3743-51.

42. Beceiro A, Llobet E, Aranda J, Bengoechea JA, Doumith M, Hornsey M, et al. Phosphoethanolamine modification of lipid A in colistin-resistant variants of Acinetobacter baumannii mediated by the pmrAB two-component regulatory system. Antimicrob Agents Chemother. 2011:55(7):3370-9.

43. Chen HD, Groisman EA. The biology of the PmrA/PmrB two-component system: the major regulator of lipopolysaccharide modifications. Annu Rev Microbiol. 2013;67:83-112.

44. Wright MS, Stockwell TB, Beck E, Busam DA, Bajaksouzian S, Jacobs MR, et al. SISPA-Seq for rapid whole genome surveys of bacterial isolates. Infect Genet Evol. 2015:32:191-8.

45. Chan AP, Sutton G, DePew J, Krishnakumar R, Choi Y, Huang XZ, et al. A novel method of consensus pan-chromosome assembly and large-scale comparative analysis reveal the highly flexible pan-genome of Acinetobacter baumannii. Genome Biol. 2015;16:143

46. Snitkin ES, Zelazny AM, Montero Cl, Stock F, Mijares L, Program NCS, et al. Genome-wide recombination drives diversification of epidemic strains of Acinetobacter baumannii. Proc Natl Acad Sci U S A. 2011;108(33):13758-63.

47. Lesho E, Yoon EJ, McGann P, Snesrud E, Kwak Y, Milillo M, et al. Emergence of colistin-resistance in extremely drug-resistant Acinetobacter baumannii containing a novel pmrCAB operon during colistin therapy of wound infections. J Infect Dis. 2013;208(7):1142-51.

48. Snitkin ES, Zelazny AM, Gupta J, Program NCS, Palmore TN, Murray PR, et al. Genomic insights into the fate of colistin resistance and Acinetobacter baumannii during patient treatment. Genome Res. 2013;23(7):1155-62.

49. Moffatt JH, Harper M, Harrison P, Hale JD, Vinogradov E, Seemann T, et al. Colistin resistance in Acinetobacter baumannii is mediated by complete loss of lipopolysaccharide production. Antimicrob Agents Chemother. 2010;54(12):4971-7.

50. Beceiro A, Moreno A, Fernandez N, Vallejo JA, Aranda J, Adler B, et al. Biological cost of different mechanisms of colistin resistance and their impact on virulence in Acinetobacter baumannii. Antimicrob Agents Chemother. 2014;58(1):518-26.

51. Marchand I, Damier-Piolle L, Courvalin P, Lambert T. Expression of the RND-type efflux pump AdeABC in Acinetobacter baumannii is regulated by the AdeRS twocomponent system. Antimicrob Agents Chemother. 2004;48(9):3298-304.

52. Ruzin A, Keeney D, Bradford PA. AdeABC multidrug efflux pump is associated with decreased susceptibility to tigecycline in Acinetobacter calcoaceticus-Acinetobacter baumannii complex. J Antimicrob Chemother. 2007;59(5):1001-4 
53. Montana S, Vilacoba E, Traglia GM, Almuzara M, Pennini M, Fernandez A, et al. Genetic variability of AdeRS two-component system associated with tigecycline resistance in XDR-Acinetobacter baumannii isolates. Curr Microbiol. 2015;71(1):76-82.

54. McCammon MT, Gillette JS, Thomas DP, Ramaswamy SV, Graviss EA, Kreiswirth $B N$, et al. Detection of rpoB mutations associated with rifampin resistance in Mycobacterium tuberculosis using denaturing gradient gel electrophoresis. Antimicrob Agents Chemother. 2005;49(6):2200-9.

55. Grangeasse C, Nessler S, Mijakovic I. Bacterial tyrosine kinases: evolution, biological function and structural insights. Philos Trans R Soc Lond B Biol Sci. 2012;367(1602):2640-55.

56. Paiment A, Hocking J, Whitfield C. Impact of phosphorylation of specific residues in the tyrosine autokinase, $\mathrm{WzC}$, on its activity in assembly of group 1 capsules in Escherichia coli. J Bacteriol. 2002;184(23):6437-47.

57. Shi L, Ji B, Kolar-Znika L, Boskovic A, Jadeau F, Combet C, et al. Evolution of bacterial protein-tyrosine kinases and their relaxed specificity toward substrates. Genome Biol Evol. 2014:6(4):800-17.

58. Halachev MR, Chan JZ, Constantinidou Cl, Cumley N, Bradley C, Smith-Banks $\mathrm{M}$, et al. Genomic epidemiology of a protracted hospital outbreak caused by multidrug-resistant in Birmingham, England. Genome Med. 2014;6(11):70.

59. Marvig RL, Sommer LM, Molin S, Johansen HK. Convergent evolution and adaptation of Pseudomonas aeruginosa within patients with cystic fibrosis. Nat Genet. 2015;47(1):57-64.

60. Diancourt L, Passet V, Nemec A, Dijkshoorn L, Brisse S. The population structure of Acinetobacter baumannii: expanding multiresistant clones from an ancestral susceptible genetic pool. PLoS One. 2010;5(4):e10034

\section{Submit your next manuscript to BioMed Central and we will help you at every step:}

- We accept pre-submission inquiries

- Our selector tool helps you to find the most relevant journal

- We provide round the clock customer support

- Convenient online submission

- Thorough peer review

- Inclusion in PubMed and all major indexing services

- Maximum visibility for your research

Submit your manuscript at www.biomedcentral.com/submit

C) Biomed Central 